\title{
A Novel Modified Transoral Approach for Endoscopic Thyroidectomy
}

\author{
Prem Kumar Anandan* \\ Bangalore Medical College and Research Institute, Bengaluru, India
}

*Corresponding Author: Dr.Prem Kumar Anandan, Bangalore Medical College and Research Institute, Bengaluru,India.Email:drpremk512@yahoo.co.in

\begin{abstract}
Background: A problem faced during totally transoral endoscopic thyroidectomy is the maneuverability of instruments due to the mandible and teeth. Swording of instruments is another hurdle in the totally transoral endoscopic thyroidectomy. These can be overcome by the modified transoral endoscopic thyroidectomy.
\end{abstract}

Methods: A $10 \mathrm{~mm}$ incision placed in the inferior vestibule in midline. A working port of $5 \mathrm{~mm}$ diameter was inserted onboth sides at the junction of upper $1 / 3^{\text {rd }}$ to lower $2 / 3^{\text {rd }}$ of the sternocleidomastoid muscle, along the anterior border. Endoscopic thyroidectomy was continued as per standard procedure.

Results: Totally eight patients underwent the modified transoral endocopic thyroidectomy. Of the eight patients, seven patients underwent hemithyroidectomy, total thyroidectomy or isthmectomy successfully as indicated. One patient was converted to conventional thyroidectomy due to inability to maintain insufflation as one of the neck ports got lacerated larger than necessary, breaching the airtight compartment.

Conclusion: The modified transoral approach is an effective and practical approach to treating solitary thyroid nodules and multinodular goiters. It provides the advantage of triangulation of instruments, while not compromising on cosmetic outcome. Though the utility of this approach in thyroid cancers with or without lymph nodal involvement needs to be studied, this approach seems promising.

Keywords: Ergonomics; Minimally Invasive Surgery; Minimally Invasive Thyroidectomy

\section{INTRODUCTION}

Theodor Billroth and Theodor Kocher, the two surgeon-physiologists, revolutionized thyroid surgery. They performed thousands of surgeries with successful results. Kocher was able to reduce the mortality associated with thyroid surgery from 1 in 6 to less than 1 in $500 .{ }^{[1]}$ Kocher was awarded Nobel Prize in 1909 for his pioneering work in thyroid physiology pathology and thyroid surgery. ${ }^{[2]}$ but thyroid surgery has remained stagnant since then. Very few innovations and techniques have been introduced since the times of Kocher.

The advent of minimally invasive surgery in the $20^{\text {th }}$ century changed the outlook of surgeons towards morbid surgeries. The focus shifted towards early recovery, minimal pain, shorter hospital stay, and cosmetic outcome.

The relative delay in the inception of endoscopic thyroidectomy could be explained by narrow operative field and numerous vital structures. The first endoscopic surgery in the neck was performed by Gagner et al in 1996 for parathyroidectomy. ${ }^{[3]}$ The first endoscopic thyroidectomy was done in 1997 by Huscher et al, in Italy. ${ }^{[4]}$

Since minimally invasive thyroid surgery was introduced in 1997, different techniques have been described: semi-open or partially endoscopic minimal neck incision, video assisted thyroidectomy and endoscopic thyroidectomy.

Wilhem and Metzeg ${ }^{[5]}$ developed a total transoral endoscopic thyroidectomy, by using a three point approach sublingually and bilaterally in the vestibule of the mouth and they made the first successful application in humans. A problem faced during totally transoral endoscopic thyroidectomy is the manoeuvrability of instruments due the mandible, alveoli and teeth.

This study aims to introduce a novel modified approach to the transoral endoscopic thyroidectomy by overcoming hurdles such as swording of instruments. It also hopes to 
overcome difficulties of instrument maneuverability.

\subsection{Objectives}

To develop a modification to the totally transoral endoscopic thyroidectomy, that is surgeon and patient friendly.

\section{METHODS}

The study period was from Jan 2016 to October 2016.The place of study was Victoria hospital, Bangalore. In this study, inclusion criteria were solitary nodule thyroid or multinodular goiter less than $5 \mathrm{~cm}$ in size, benign disease on fine needle aspiration biopsy. The exclusion criteria were large nodules larger than $5 \mathrm{~cm}$, malignant features on fine needle aspiration biopsy, and patients who did not consent to the surgery.

Patients undergoing modified transoral minimally invasive thyroidectomy were given chlorhexidine mouth wash twice daily for 3 days preoperatively. Patients were placed in supine position with extension of the neck supported by a head ring and shoulder elevation by using sand bag (Figure 1).Patients were anaesthetized by nasotracheal intubation.

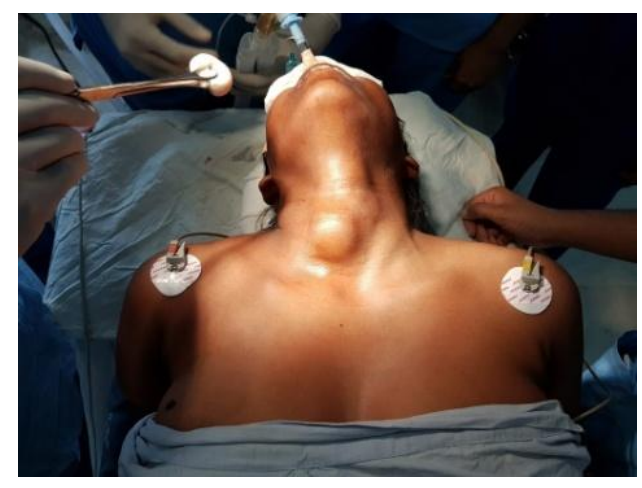

Figure1. Position of the patient

Surgeon and assistant were positioned at the head end. Monitor was placed on the side of the patient. Sternal notch and skin overlying the nodule were marked using skin pencil. Oral cavity was painted using normal saline and throat was packed. $2 \%$ lignocaine with adrenaline diluted to $1: 20$, was infiltrated along the plane of dissection from the vestibule till neck. A $10 \mathrm{~mm}$ incision was placed in the inferior vestibule in midline. A $10 \mathrm{~mm}$ port was inserted through this incision in the subcutaneous plane into the neck by blunt dissection. Carbon dioxide insufflation was given with pressure set at $7-8 \mathrm{~mm}$ of $\mathrm{Hg}$. Subplatysmal plane was created deep to deep fascia. 30 degree scope of diameter $10 \mathrm{~mm}$ was passed through incision. The structures were visualized in craniocaudal view.

A working port of $5 \mathrm{~mm}$ diameter was inserted on both sides at the junction of upper $1 / 3^{\text {rd }}$ to lower $2 / 3^{\text {rd }}$ of the sternocleidomastoid muscle, along the anterior border (Figure 2 and 3). Ultrasonic harmonic scalpel was inserted through the working ports and medial fibres of strap muscles covering the thyroid gland were divided, along with the loose areolar tissue for better visualization of the gland. Superior pole of the thyroid gland was dissected and divided using harmonic scalpel. Toothed or non-toothed forceps were inserted and the gland held with it. The gland was retracted and dissection carried out using harmonic scalpel to release the gland from prevertebral fascia, trachea and the other adjacent structures. Lower pole of the gland was divided. The isthmus was also divided in similar fashion from trachea. Thyroid gland was divided at the junction of isthmus and the contralateral lobe using harmonic scalpel in cases where hemithyroidectomy was done.

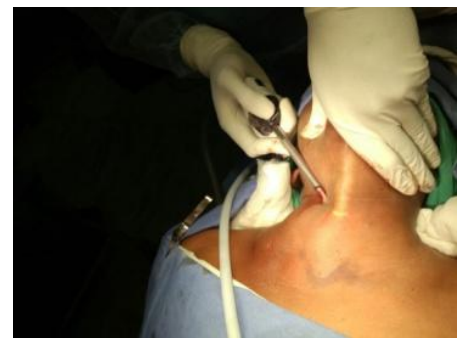

Figure2. Insertion of working port at the junction of upper one third and lower two thirds of the sternocleidomastoid muscle at the anterior border

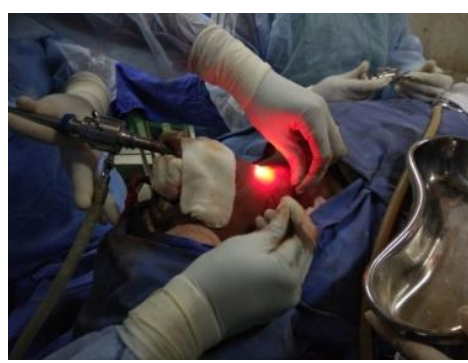

Figure3. Insertion of working port. Scope is seen in the vestibule.

Recurrent laryngeal nerves were identified and saved. Parathyroid glands were visualized and saved. The specimen was retrieved through one of the neck ports. Neck incisionwas minimally extended only when required to extract the specimen. Absolute haemostasis was achieved using harmonic. A suction drain was placed and brought out through the vestibular port. The camera was withdrawn after confirming haemostasis. $\mathrm{CO}_{2}$ used for insufflation was released. 
Incision over the vestibule was closed with simple sutures using polygalactin. The port sites in the neck were sutured in layers. Throat pack removed and sterile dressing placed. Movement of vocal cords noted at the time of extubation.

The patients were monitored post-operatively for haemorrhage, recurrent laryngeal nerve injury and hypocalcemia. The drain was removed when $24 \mathrm{hrs}$ drainage was less than $30 \mathrm{ml}$.

Patients were given IV antibiotics for 3days.Allowed orally from post-operative day1.Continued chlorhexidine mouth wash for one week. Sutures removed on seventh postoperative day. Patients were followed up for signs of hypocalcemia and surgical site infections.

\section{RESULTS}

A total of eight patients underwent the modified transoral endocopic thyroidectomy. Six patients were female and two were male. Youngest patient was 28 years of age and the oldest was 80 years old, with a mean age of 50 years with a standard deviation of 14.39. Of the eight patients, three patients with multinodular goiter underwent total thyroidectomy. One patient underwent isthmectomy for solitary nodule over the isthmus. Two patients had a solitary nodule in the left lobe of the thyroid, while two had a solitary nodule in the right lobe. Three of these patients with solitary nodule sumlessfully underwent hemithyroidectomies. One of these patients was converted to conventional thyroidectomy due to inability to maintain insufflation as one of the neck ports got lacerated larger than necessary, therefore breaching the airtight compartment.

All solitary nodules on fine needle aspiration biopsy were diagnosed as colloid adenoma. All three multinodular goiters were diagnosed as colloid nodular goiter with cystic change. Patients chose surgery in view of cosmetic appearance.

The mean operating time was 118.75 minutes with a standard deviation of 15.52 minutes.

The first attempt at this approach took 150 minutes, while the last case took 100 minutes.

Blood loss was less than $10 \mathrm{ml}$ in each case. No patient developed recurrent or superior laryngeal nerve injury, as confirmed by visualization of the vocal cords at extubation, and absence of voice changes. No patient developed postoperative hypocalcemia. Both neck ports and the vestibular port healed by primary intention in all patients (Figure 4 and 5).One patient developed an abscess over the anterior aspect of the neck in the midline, which was drained and treated with antibiotics as per pus culture and sensitivity. Out of the eight patients, one patient was converted to conventional thyroidectomy, as mentioned above.

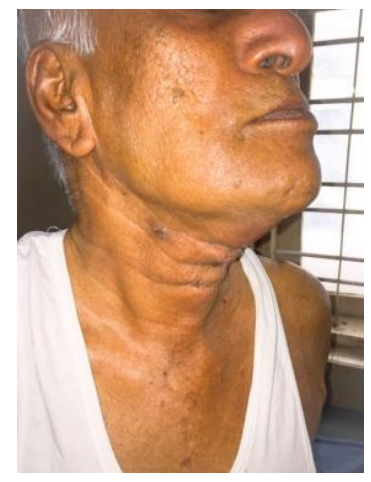

Figure4. Post-operative picture of neck port

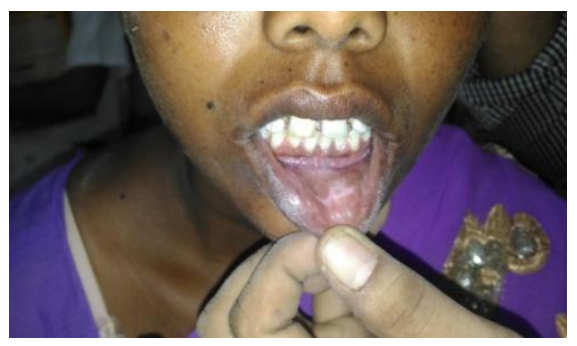

Figure5. Post-operative picture of vestibular port

\section{DISCUSSION}

In our study, we modified the total transoral approach. The camera port was retained in the oral vestibule whereas the working $5 \mathrm{~mm}$ ports were placed high in the neck. The neck ports were placed high in the neck so that they not visible when the patient is looking forward. One of the neck ports was used for drain placement.

Ergonomics plays a very important role in minimally invasive surgeries. It is well known amongst minimally invasive surgeons, that ergonomically well designed instruments and orientation of instruments plays a key role in the outcome of surgery. ${ }^{[6]}$ A key hurdle faced in the totally transoral technique is the swording of instruments. The extreme acute angle between the working instruments causes technical difficulty in maneuvering them. Obstruction by the mandible is another obstacle. In this regard, our modification increases the angle between the instruments to 60-90 degrees, as opposed to 0-5 degrees in the totally transoral technique, making it surgeon friendly by creating triangulation of the instruments (Figure 6). ${ }^{[7]}$ 
The improved ergonomics is the key feature of the modified transoral approach.

Another key advantage of this modification is the cosmetically favourable post-operative result. Though this modification is not "scarless" as in the totally transoral approach, it has the pros of ergonomics and maneuverability which greatly outweigh the minor cons of cosmesis.

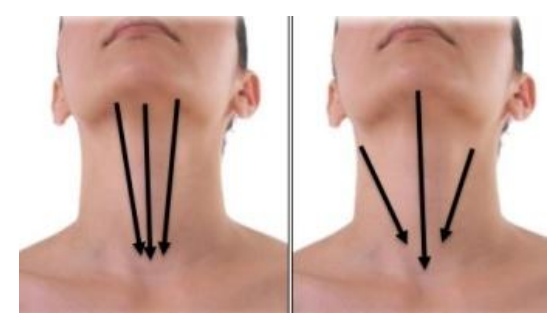

Figure6. Depiction of swording in totally transoral endoscopic thyroidectomy, as opposed to triangulation of modified transoral endoscopic thyroidectomy

A precaution that must be taken in our modified approach is regarding the working ports. Care must be taken to ensure the incision for the port is not too large, else, carbon dioxide insufflation will fail. Unlike the abdomen, the neck does not possess a strong muscular layer to ensure insufflation remains intact.

Minimally invasive thyroid surgery will be greatly benefitted with advancement in the surgical instruments. Better instruments will result in greater efficiency and hence, better outcomes. ${ }^{[8]}$

\section{CONCLUSION}

The modified transoral approach is an effective and practical approach to treating solitary thyroid nodules, and multinodular goiters. It provides the advantage of triangulation of instruments, while not compromising on cosmetic outcome. Though the utility of this approach in thyroid cancers with or without lymph nodal involvement needs to be studied, this approach seems promising.

\section{REFERENCES}

[1] Lal G, Clark OH. Thyroid, Parathyroid and Adrenal. In: Brunicardi F C, Anderson D K, Billiar T R, Dunn D L, Hunter J G, Matthews J B, Pollock R E, editors. Schwartz's Principles of Surgery. $10^{\text {th }}$ ed. McGraw Hill Education; 2015.

[2] Smith PW, Hanks LR, Solomone LJ, Hanks JB. Thyroid. In: Townsend CM, Beauchamp RD, Evers BM, Mattox KL, editors. Sabiston Textbook of Surgery. $20^{\text {th }}$ ed. Elsevier; 2017.

[3] Gagner M. Endoscopic subtotal parathyroidectomy in patients with primary hyperparathyroidism. Br J Surg. 1996 Jun; 83(6): 875.

[4] Huscher CS, Chiodini S, Napolitano C, Reacher A. Endoscopic right thyroid lobectomy. Surg Endosc.1997;11;(8):877.

[5] Wilhem T, Metzig A. Endoscopic minimally invasive thyroidectomy: first clinical experience. Surg Endosc. 2010;24(7):1757-8.

[6] Manasnayakorn S, Cuschieri A, Hanna GB. Ideal manipulation angle and instrument length in hand-assisted laparoscopic surgery. Surg Endosc. 2008;22:924-9.

[7] Trejo A, Jung MC, Oleynikov D, Hallbeck MS. Effect of handle design and target location on the insertion and aim with a laparoscopic surgical tool. Appl Ergon. 2007;38:745-53.

[8] Falk V, McLoughlin J, Guthart G, Salisbury JK, Walther T, Gummert J, et al. Dexterity enhancement in endoscopic surgery by a computer-controlled mechanical wrist. Minim Invasive Ther Allied Technol. 1999;4:235-42.

Citation: Prem Kumar Anandan. A Novel Modified Transoral Approach for Endoscopic Thyroidectomy. ARC Journal of Surgery.2019; 5(2):4-7.doi:dx.doi.org/10.20431/2455-572X. 0405002.

Copyright: (C) 2019 Authors. This is an open-access article distributed under the terms of the Creative Commons Attribution License, which permits unrestricted use, distribution, and reproduction in any medium, provided the original author and source are credited. 